\title{
Oral treatment with herbal formula B40I alleviates penile toxicity in aging mice with manganism
}

This article was published in the following Dove Press journal:

Clinical Interventions in Aging

28 May 2015

Number of times this article has been viewed

\author{
Chih-Hsiang Hsu' \\ Ching-Lung Lin' \\ Sheue-Er Wang' \\ Shuenn-Jyi Sheu ${ }^{2}$ \\ Chiang-Ting Chien' \\ Chung-Hsin $\mathrm{Wu}^{\prime}$ \\ 'Department of Life Science, National \\ Taiwan Normal University, ${ }^{2}$ Brion \\ Research Institute of Taiwan, Taipei, \\ Taiwan
}

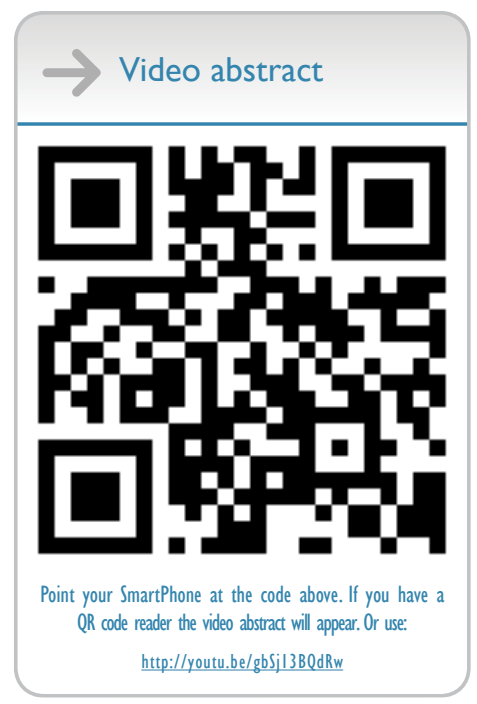

Correspondence: Chung-Hsin Wu Department of Life Science, National Taiwan Normal University, 88, Ting-Chou Road, Section 4, Taipei II677, Taiwan

Tel +8862 77346363

Fax +886 2 293I 2904

Email megawu@ntnu.edu.tw
Abstract: The present study aims to elucidate the roles of nitric oxide synthase activity, oxidative stress, inflammation, and apoptosis in penile toxicity of aging mice associated with excess manganese $(\mathrm{Mn})$ treatment and to investigate the effect of oral treatment with the herbal formula B401 in this respect. ICR strain mice were divided into two groups: the vehicle (sham group) and the B401 (50 mg/kg) group. The mice were orally treated for 5 days; then a high single dose of $\mathrm{MnCl}_{2}(100 \mathrm{mg} / \mathrm{kg})$ was given by intraperitoneal injection to the mice. One day after $\mathrm{MnCl}_{2}$ treatment, corpora cavernosal tissues of both Mn-treated mice and their controls were simultaneously sampled to examine their immunohistochemical staining and Western blot analysis. Nitric oxide (NO) production, levels of neuronal nitric oxide synthase (nNOS) and endothelial nitric oxide synthase (eNOS), expression levels of factors governing angiogenesis (vascular endothelial growth factor), oxidative stress (catalase, superoxide dismutase 2,4-hydroxynonenal), inflammation (tumor necrosis factor alpha), apoptosis (B-cell lymphoma 2 [Bcl-2], Bcl-2-associated X protein [Bax], cleaved poly(adenosine diphosphateribose) polymerase [c-PARP], cytochrome $\mathrm{C}$, caspase-12, and caspase-3) were evaluated in penile corpus cavernosum of the mice. We found that penile toxicity in the mice was enhanced under excess Mn treatment through reduction of NOS activity and increase in oxidative stress, inflammation, and apoptosis in the penile cavernous tissue. Furthermore, the penile toxicity in mice with manganism was alleviated by oral B401 treatment through enhancement of both nitric oxide synthesis and angiogenesis, with simultaneous reduction of oxidative stress, inflammation, and apoptosis in penile corpus cavernosum. We suggest that the herbal formula B401 may serve as a potential dietotherapeutic supplement for penile toxicity or dysfunction in aging males.

Keywords: manganism, herbal formula, oxidative stress, inflammation, apoptosis

\section{Introduction}

From clinical case reports, welders often experience problems with impotence when they are occupationally exposed to excess manganese (Mn) dust. ${ }^{1-3}$ In addition, male workers may show reduced fertility when exposed to Mn dust. ${ }^{4}$ In animal experiments, male rabbits had often shown testicular degeneration and sterility when exposed to a high dose of Mn. ${ }^{5,6}$ Until now, possible mechanisms for Mn-induced reproductive toxicology have rarely been explored. Occupational exposure to high levels of Mn can cause a syndrome known as manganism, which has symptoms that closely resemble Parkinson's disease. In this study, we developed an aged mouse model of manganism in penile toxicity.

Activity of nitric oxide synthase (NOS) in the corpus cavernosum plays a key role in penile erection. ${ }^{7,8}$ In the penile tissue, nitric oxide is released in the corpus cavernosum by regional expression of neuronal, inducible, and endothelial NOSs (nNOS, iNOS, and eNOS, respectively). ${ }^{9,10}$ Reduction of nitric oxide release in penile tissue 
was often observed with aging, as well as in specific diseases such as diabetes mellitus, hyperlipidemia, and hypertension, which often cause erectile dysfunction. ${ }^{11-13}$ In addition, oxidative stress and downregulation of vascular endothelial growth factor (VEGF) expression were often observed in the corpus cavernosum of impotent animals. ${ }^{14-16}$ Oxidative stress and a VEGF deficit may be involved in manganism-induced reproductive toxicology.

Recently, the Brion Research Institute of Taiwan has developed the herbal formula B401 for use as an alternative health supplement. It has been suggested that the herbal formula B401 can be developed as a dietotherapeutic supplement for ameliorating neurodegenerative diseases such as Huntington's disease (HD) by enhancing VEGF and brain-derived neurotrophic factor, while reducing inflammation in the brain tissue. ${ }^{17}$ In addition, we also found that the herbal formula B401 has neuroprotective effects in HD transgenic mice by reducing oxidative stress and apoptosis. We reasonably infer that the herbal formula B401 may be a potential dietotherapeutic supplement for ameliorating manganism-induced reproductive toxicology in mice through enhancement of NOS activity, as well as reduction of oxidative stress, inflammation, and apoptosis signaling pathways. In this study, we elucidated the role of NOS activity, oxidative stress, inflammation, and apoptosis in penile toxicity of ICR mice associated with Mn and investigated the effect of oral treatment with the herbal formula B401 in this respect.

\section{Materials and methods}

\section{Chromatographic fingerprint analysis for the herbal formula B40I}

The herbal formula B401 has a Taiwan-US patent (\#US 7,838,048 B2). It contains six herbal ingredients, including extracts from Panax ginseng, Astragalus membranaceus, Angelica sinensis, Rehmannia glutinosa, Ligustri Fructus, and Eclipta prostrata in specific ratios, and has been developed as a health supplement. High-performance liquid chromatography (HPLC) charts of the six marker compounds (ginsenosides Rb1, formononetin, 5-hydroxymethylfurfural, ferulic acid, oleanolic acid, and wedelolactone) of the herbal formula B401 with different wavelengths are shown in Figure 1. All compounds used in this analysis were solubilized in distilled $\mathrm{H}_{2} \mathrm{O} / \mathrm{MeOH}$. HPLC-grade acetonitrile from Burdick and Jackson (Gyeonggi-do, Korea) and methanol from Avantor (Center Valley, PA, USA) were used with these herbal ingredients. A Milli-Q water purification system (EMD Millipore, Billerica, MA, USA) provided purified water.
For further information, experiments would not be affected by the vehicle.

\section{Animal preparation and monitoring}

In this study, a total of 18 ICR mice at 24 months of age were divided into the sham group $(n=6)$, Mn group $(n=6)$, and the B401+Mn group $(n=6)$. The mice of the sham group were fed with the same amount of distilled water without B401 and Mn treatment. Those of the Mn group were given an intraperitoneal injection with a high single dose of manganese chloride $\left(\mathrm{MnCl}_{2}, 100 \mathrm{mg} / \mathrm{kg}\right.$, the $\mathrm{pH}$ value was close to 7.0) without oral B401 treatment. Mice of the B401+Mn group were orally fed with the B401 formulation $(50 \mathrm{mg} / \mathrm{kg}$, the $\mathrm{pH}$ value was close to 7.0) twice every day for 5 days and then treated with a high single dose of $\mathrm{MnCl}_{2}(100 \mathrm{mg} / \mathrm{kg}$, the $\mathrm{pH}$ value was close to 7.0) by intraperitoneal injection. All doses of the herbal formula B401 were adjusted according to individual weight and water consumption, which was much lower than the dosage of half-maximal inhibitory concentration $\left(\mathrm{IC}_{50}\right)$. One day after $\mathrm{MnCl}_{2}$ treatment, the levels of cavernosal nitric oxide in the mice of the Mn group and B401+Mn group were examined with a nitric oxide electrochemical sensor meter. The level of cavernosal nitric oxide in the mice of the sham group was also examined at the same time. All protocols in the experiment were executed according to the international guidelines for care and use of laboratory animals. This animal experiment and drug trials on animals was approved by the Committee on Animal Research of National Taiwan Normal University (NTNU) and implemented under the guidelines of the Committee (protocol number: NTNU/ Animal Use and Care/No 103017).

\section{Measurements of cavernosal nitric oxide in the penile tissue}

One day after $\mathrm{MnCl}_{2}$ treatment, the expression of cavernosal nitric oxide in the penile tissue of the mice was measured with a nitric oxide electrochemical sensor meter (Model IMEC-601; Inter Medical Co, Tokyo, Japan). As in a previous study, ${ }^{18}$ the nitric oxide-selective microelectrode was calibrated by the polarographic spectrum. The counter electrode was made of carbon fiber (outer diameter [OD]: $0.5 \mathrm{~mm}$ ) and surrounded the working electrode. The working electrode consisted of a platinum/iridium alloy wire (OD: $0.2 \mathrm{~mm}$ ) coated with a three-layer membrane that included $\mathrm{KCl}$ resin, a nitric oxide-selective membrane, and a normal silicone membrane. The electrode touched the surface of the penis, and the tip of the electrode was positioned in the middorsal artery. Electrochemical oxidation of nitric 


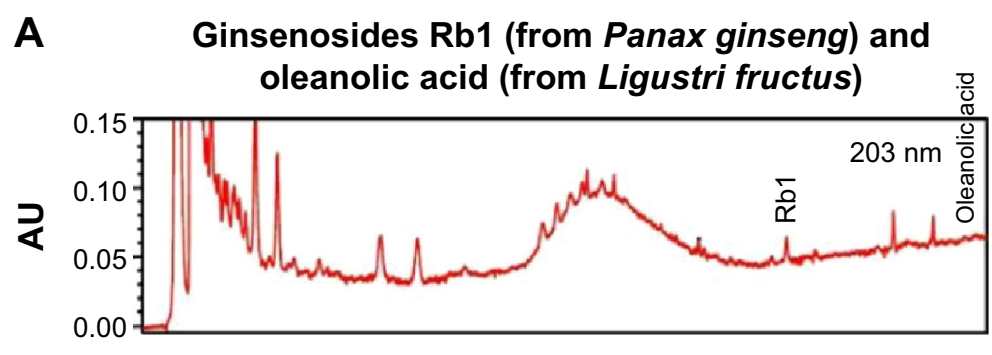

\section{B Formononetin (from Astragalus membranaceus)}
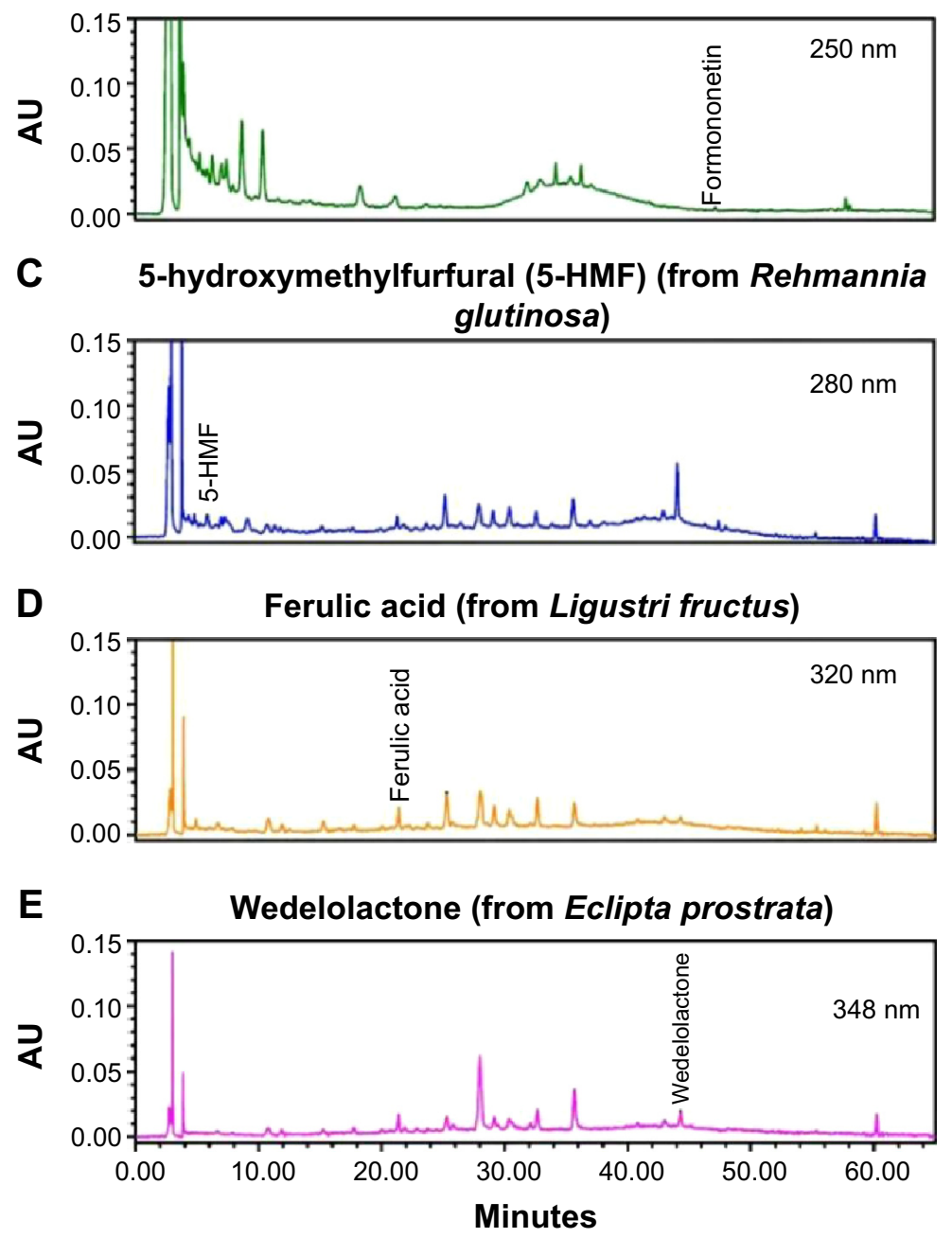

Figure I Chromatographic fingerprint analysis for the Chinese herbal formula B40I.

Notes: HPLC fingerprint of the herbal formula B40I. Characteristic peaks of B40I, ie, A: ginsenosides Rb I (C54H92O23; MW: I l09.3 g/mol; from Panax ginseng) and oleanolic acid (C30H48O3; MW: $456.7 \mathrm{~g} / \mathrm{mol}$; from Ligustri fructus); B: formononetin (Cl6HI2O4; MW: $268.3 \mathrm{~g} / \mathrm{mol}$; from Astragalus membranaceus); C: 5-hydroxymethylfurfural (C6H6O3; MW: $126.1 \mathrm{~g} / \mathrm{mol}$; from Rehmannia glutinosa); D: ferulic acid (Cl0HI0O4; MW: $194.2 \mathrm{~g} / \mathrm{mol}$; from Ligustri fructus); E: wedelolactone (Cl6HI0O7; MW: 3I4.3 g/mol; from Eclipta prostrata), were identified and marked at the corresponding peaks in the fingerprint.

Abbreviations: AU, arbitrary perfusion units; 5-HMF, 5-hydroxymethylfurfural; HPLC, high-performance liquid chromatography; MW, molecular weight.

oxide at the electrode surface was measured by the current polarographic electrode. The output current from the electrodes were monitored on the monitor and were recorded in the LabChart (AD Instrument Pty Ltd, New South Wales, Australia). The change percentage provided an index of changes in tissue nitric oxide concentrations on the basis of the changes in output currents.

\section{Immunohistochemical stain for eNOS and $\mathrm{nNOS}$ protein expression}

One day after $\mathrm{MnCl}_{2}$ treatment, corpora cavernosal tissues of both Mn-treated mice and their controls were simultaneously sampled to examine their immunohistochemical (IHC) staining. After formalin fixing, the cavernosal tissue section $(5 \mu \mathrm{m})$ immersed in citrate buffer was applied to a 
heart-induced epitope retrieval for 15 minutes; it was next reacted with the primary antibody. Mouse anti-eNOS (1:200) (Cell Signaling Technology Inc, Danvers, MA, USA) binds to eNOS as a primary antibody, and rabbit anti-nNOS (1:500) (Cell Signaling Technology Inc). The slide was kept at room temperature for 60 minutes. The slide was washed with phosphate-buffered saline (PBS) for 10 minutes, reacted with the secondary antibody attached with biotin for 30 minutes, and then reacted with the chromogen 3,3'-diaminobenzidine tetrahydrochloride for 5 minutes. The slide was then reacted with NoVolink polymer (Leica, polymer detection system) for 30 minutes, and it was washed with PBS three times. The IHC staining was examined by one researcher.

\section{Western blot analysis}

One day after $\mathrm{MnCl}_{2}$ treatment, the corpora cavernosal tissues of Mn-treated mice and their controls were simultaneously sampled to obtain their Western blots. The removed penile tissue was homogenized in a buffer solution that included $0.05 \mathrm{M}$ tris (hydroxymethyl) aminomethane (Tris, $\mathrm{pH} 8.0$ ), $0.15 \mathrm{M}$ sodium chloride, $0.02 \mathrm{M}$ ethylenediaminetetraacetic acid, 1\% deoxycholic acid, 1\% nonidet P40, 0.1\% sodium dodecyl sulfate (SDS), 1\% protease inhibitor cocktail for full range, $1 \%$ serine/threonine phosphatase inhibitor cocktail, and $1 \%$ tyrosine phosphatase inhibitor cocktail (all from Bionovas, Washington, DC, USA). The homogenized buffer solution was placed on ice for 1 hour, centrifuged at $4^{\circ} \mathrm{C}$ for 13,000 rpm for another 20 minutes, and the supernatant solution was separated. The separated solution was quantitated by using a bicinchoninic acid protein assay kit (Thermo Fisher Scientific Inc, Waltham, MA, USA). Thirty micrograms of the total protein was denatured at $95^{\circ} \mathrm{C}$ for 5 minutes with $5 \times$ sample dye, which included $0.25 \mathrm{M}$ Tris- $\mathrm{HCl}$ (pH 6.8, Bionovas), 10\% SDS (Bionovas), 0.5\% bromophenol blue (Bionovas), 50\% glycerol (Bionovas), and 5\% beta-mercaptoethanol (Bionovas). The electrophoresis was performed with $12.5 \%$ discontinuous SDS-polyacrylamide gel. The proteins were then electroblotted onto a $0.2 \mu \mathrm{m}$ polyvinylidenedifluoride membrane (GE Healthcare Life Sciences, Barrington, IL, USA) for 120 minutes at $100 \mathrm{~V}$. The membranes were reacted with a blocking buffer $(5 \%$ skimmed milk in Tris-buffered saline and Tween 20 [TBS-T] buffer) for 1 hour at ambient temperature, and then they were blocked.

The antibodies to 4-hydroxynonenal (4-HNE), cytochrome C (Cyt-C; Abcam, Cambridge, MA, USA), alphatubulin, beta-actin, Bcl-2-associated $\mathrm{X}$ protein (Bax; Thermo Fisher Scientific Inc), B-cell lymphoma 2 (Bcl-2;
Santa Cruz Biotechnology Inc, Santa Cruz, CA, USA), catalase, caspase-12, caspase-3, eNOS, nNOS, cleaved poly(adenosine diphosphate-ribose) polymerase (c-PARP), superoxide dismutase 2 (SOD2), and tumor necrosis factor alpha (TNF- $\alpha$; Cell Signaling Technology Inc) were added and the mixtures reacted for 2 hours at ambient temperature (or overnight at $4^{\circ} \mathrm{C}$ ). The membrane was washed three times using TBS-T at intervals of 10 minutes. As the secondary antibodies, anti-rabbit immunoglobulin G (IgG)-horseradish peroxidase (HRP; 1:5,000 dilution), anti-mouse IgG-HRP (1:5,000 dilution), and anti-goat IgG-HRP (1:5,000 dilution, PerkinElmer, Waltham, MA, USA) were reacted at ambient temperature for 1 hour, and the membrane was washed again with TBS-T three times, with an interval of 10 minutes between each washing. Each band was visualized using enhanced chemiluminescence Western blotting detection reagents (GE Healthcare Life Sciences, Barrington, IL, USA), and the chemiluminescence was detected using LAS-4000 (GE Healthcare Life Sciences). Densitometric assessments of the bands were performed using ImageJ software (version 1.48t; Wayne Rasnabd, Washington, DC, USA).

\section{Statistical analysis}

All data are given as mean \pm standard error of the mean from at least six independent experiments. Statistical analysis was performed using one-way analysis of variance followed by Student-Newman-Keuls multiple comparisons posttest. $P$-values of at least $<0.05$ were considered significant.

\section{Results \\ Effect of oral B40I treatment on cavernosal synthesis of nitric oxide and angiogenesis in Mn-treated mice}

Effect of B401 treatment on cavernosal synthesis of nitric oxide is shown in Figure 2A. As detected by the electrochemical sensor meter, the value of cavernosal plateletderived nitric oxide in Mn-treated mice was significantly lower than that in the sham mice (Figure 2A(b), Mn group: $450.2 \pm 12.2 \mathrm{pA}$, vs Sham group: $477.8 \pm 14.4 \mathrm{pA}, P<0.05)$. The cavernosal nitric oxide value in Mn-treated mice under B401 treatment was significantly higher than those in Mn-treated mice without B401 treatment (Figure 2A(b), B401+Mn group: $502.2 \pm 17.8 \mathrm{pA}$ vs Mn group: $450.2 \pm 12.2 \mathrm{pA}$, $P<0.05)$. The cavernosal nitric oxide value in Mn-treated mice was reduced to $\sim 94 \%$ of the value of sham mice, but it was $\sim 105 \%$ of the value of sham mice in the Mn-treated 
A
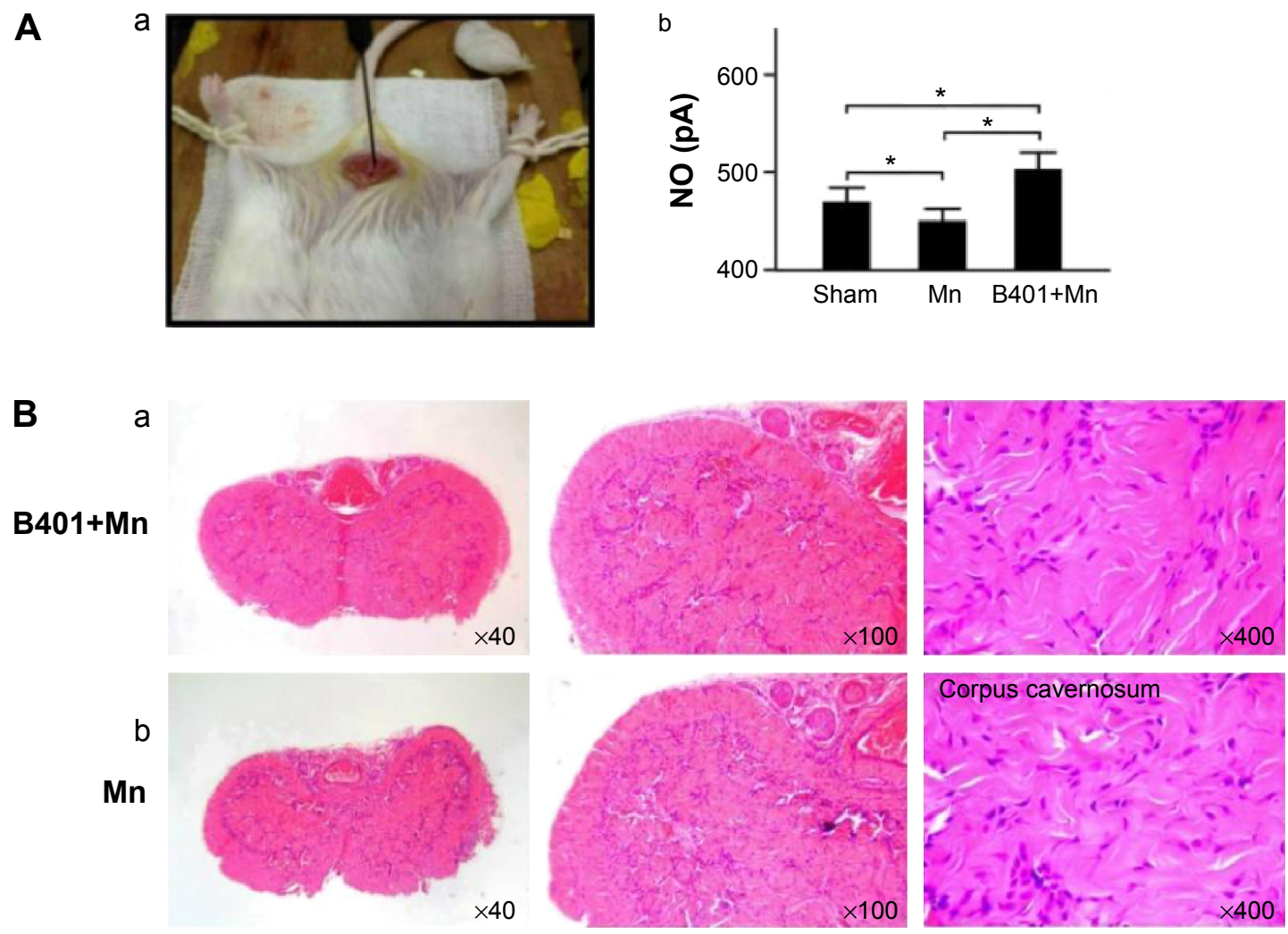

Figure 2 Cavernosal nitric oxide (NO) levels and H\&E staining in Mn-treated mice with and without oral B40I treatment.

Notes: (A) Cavernosal NO levels measured by NO electrochemical sensor meter; (a) surgical technique used for NO monitoring in vivo; and (b) quantified cavernosal NO levels in Mn-treated mice under oral B40I treatment (B40I+Mn, $n=6$ ) were significantly higher than those in Mn-treated mice without oral B40I treatment (Mn, $n=6)$. Values are mean \pm SEM $(* P<0.05$, one-way ANOVA followed by a Student-Newman-Keuls multiple comparisons posttest). (B) Cavernosal H\&E staining in (a) Mn-treated mice under oral B40I treatment (B40I+Mn) and (b) cavernosal tissue in Mn-treated mice without oral B40I treatment (Mn).

Abbreviations: ANOVA, analysis of variance; H\&E, hematoxylin and eosin; NO, nitric oxide; SEM, standard error of the mean.

mice under B401 treatment (Figure 2A(b)). Interestingly, the cavernosal nitric oxide value in the cavernosal tissues of Mntreated mice under oral B401 treatment was also significantly higher than those in the sham mice (Figure 2A(b), B401+Mn vs Sham, $P<0.05$ ). From hematoxylin-and-eosin (H\&E) histological study, we observed that cavernosal tissues in Mn-treated mice with and without oral B401 administration were very similar (Figure 2B).

The effect of B401 treatment on expression levels of cavernosal NOS is shown in Figure 3. Our IHC staining study revealed that cavernosal eNOS expression levels of Mn-treated mice under B401 treatment (B401+Mn group) were visibly enhanced beyond those of Mn-treated mice without B401 treatment (Mn group) (Figure 3A, B401+Mn vs $\mathrm{Mn})$. We quantified cavernosal eNOS expression levels of Mn-treated mice by Western blotting analysis, as shown in Figure 3B. Both cavernosal nNOS and eNOS expression levels of Mn-treated mice under B401 treatment were significantly higher than those in the Mn-treated mice without B401 treatment (Figure 3B, B401+Mn vs Mn, $P<0.01)$.
We further quantified the expression levels of cavernosal VEGF expression levels by Western blotting analysis, as shown in Figure 4A. VEGF is a marker protein that stimulates angiogenesis. We found that the expression levels of VEGF in the cavernosal tissues of Mn-treated mice were not significant when compared with mice under sham treatment (Figure 4B, Mn vs Sham, $P>0.05$ ). However, the expression levels of VEGF in the cavernosal tissues of Mn-treated mice under B401 treatment were significantly higher than those in the Mn-treated mice without B401 treatment (Figure 4B, $\mathrm{B} 401+\mathrm{Mn}$ vs $\mathrm{Mn}, P<0.01$ ). Interestingly, expression levels of VEGF in the cavernosal tissues of Mn-treated mice under oral B401 treatment were significantly higher than those in the sham mice (Figure 4B, B401+Mn vs Sham, $P<0.05$ ).

\section{Effect of oral B40I treatment on marker proteins of oxidative stress and inflammation in the cavernosal tissues of Mn-treated mice}

The effect of B401 treatment on expression levels of marker proteins of oxidative stress in the cavernosal tissues is shown 
A
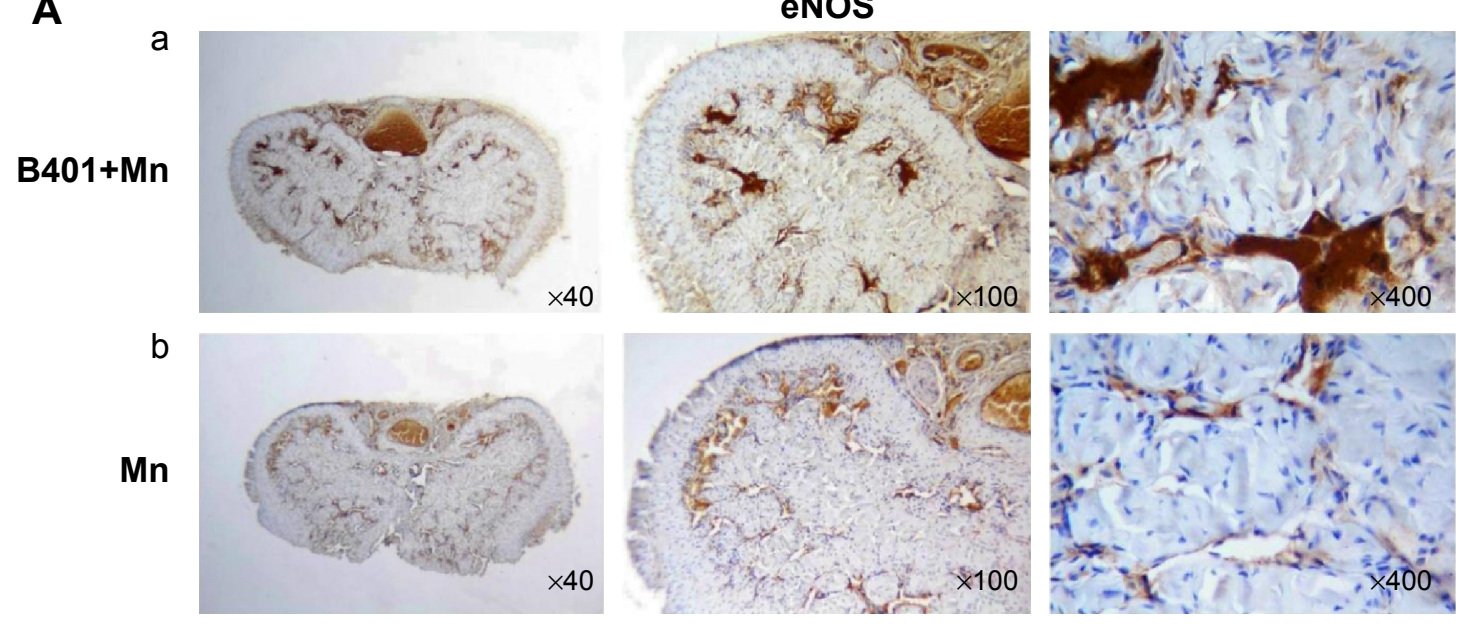

B
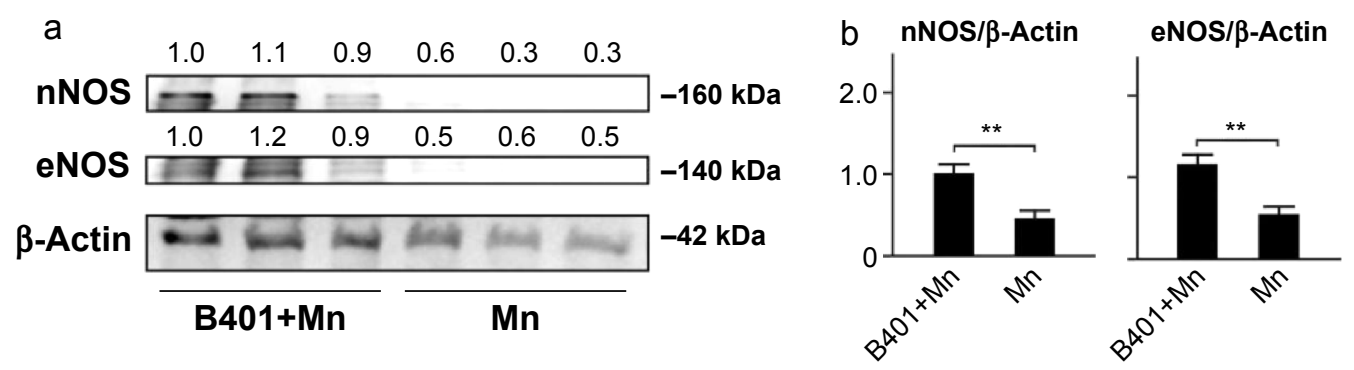

Figure 3 Cavernosal levels of nitric oxide synthases in Mn-treated mice with and without oral B40I treatment.

Notes: (A) Immunohistochemical staining illustrated that expression levels of cavernosal eNOS of (a, B40I+Mn) Mn-treated mice under oral B40I treatment were visibly enhanced in comparison with the (b, Mn) Mn-treated mice without oral B40I treatment. (B) Western blotting analysis shows the following: (a) cavernosal expression levels of $\mathrm{nNOS}$ and eNOS of Mn-treated mice with and without oral B40I treatment and (b) quantified cavernosal nNOS and eNOS levels of Mn-treated mice with oral B40I treatment $(B 40 I+M n, n=6)$ were significantly enhanced in comparison with the same in Mn-treated mice without oral B40I treatment (Mn, $n=6)$. Values are mean $\pm S E M$ ( $* * P<0.01$, one-way ANOVA followed by Student-Newman-Keuls multiple comparisons posttest).

Abbreviations: nNOS, neuronal nitric oxide synthase; eNOS, endothelial nitric oxide synthase; SEM, standard error of the mean; ANOVA, analysis of variance.

in Figures 4 and 5. We quantified the expression levels of catalase and SOD 2 by Western blotting analysis, as shown in Figure 4A. Expression levels of both catalase and SOD2 in the cavernosal tissues of Mn-treated mice were significantly weaker than those in the sham mice (Figure 4B, Mn vs Sham, $P<0.05$ ), but the levels were significantly increased in the cavernosal tissues of Mn-treated mice under oral B401 treatment (Figure 4B, B401+Mn vs Mn, $P<0.01$ ). Interestingly, expression levels of both catalase and SOD2 in the cavernosal tissues of Mn-treated mice under oral B401 treatment were significantly increased beyond those of the sham mice (Figure 4B, B401+Mn vs Sham, $P<0.01$ ). We further quantified the expression levels of 4-HNE by Western blotting analysis, as shown in Figure 5A. 4-HNE has been hypothesized to play a key role in oxidative stress by increasing the lipid peroxidation chain reaction. We found that the expression levels of 4-HNE in the cavernosal tissues of Mn-treated mice under oral B401 treatment were significantly weaker than those of the Mn-treated mice without B401 treatment (Figure 5B, B401+Mn vs Mn, $P<0.01$ ).
The effect of B401 treatment on oxidative stress in the cavernosal tissues is shown in Figure 6. We quantified the expression levels of TNF- $\alpha$ by Western blotting analysis, as shown in Figure 6A. TNF- $\alpha$ is a signaling protein that is involved in systemic inflammation. We found that the expression levels of TNF- $\alpha$ in the cavernosal tissues of Mn-treated mice under oral B401 treatment were significantly weaker than those of the Mn-treated mice without B401 treatment (Figure 6B, B401+Mn vs Mn, $P<0.01$ ).

\section{Effect of oral B40I treatment on marker proteins of apoptosis in the cavernosal tissues of Mn-treated mice}

It has been described that apoptosis is a downstream event in erectile dysfunction. ${ }^{19}$ Therefore, we examined and compared expressions of apoptosis-related proteins in cavernosal tissues of Mn-treated mice with and without oral B401 treatment (Figure 7A). As suggested in previous studies, ${ }^{20} \mathrm{Bcl}-2$ is a marker of antiapoptosis, while Bax and Cyt-C are two markers of mitochondrial apoptosis. The ratio of $\mathrm{Bcl}-2$ protein to 
A

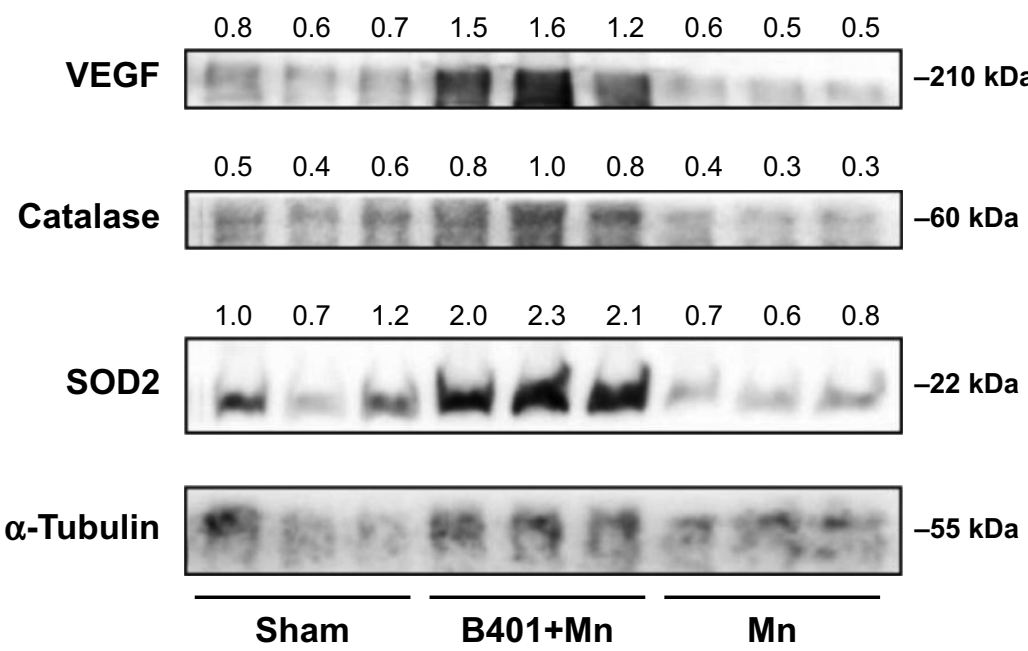

B
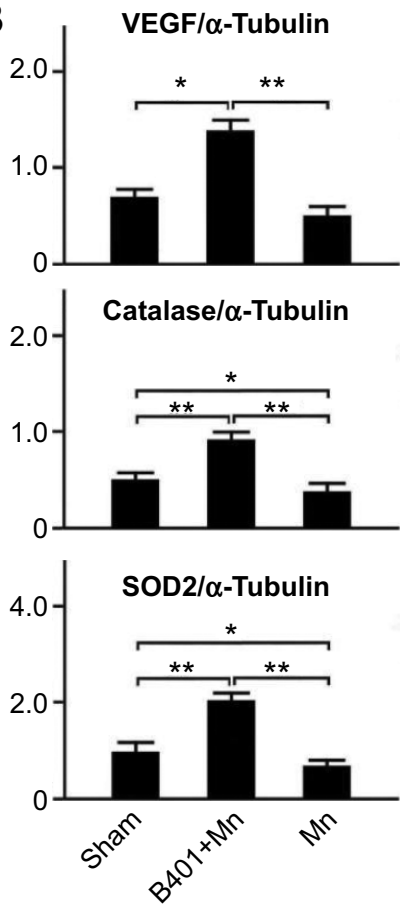

Figure 4 Cavernosal levels of VEGF, catalase, and SOD2 among Mn-treated mice with or without oral B40I treatment and among the sham mice.

Notes: Western blotting analysis shows the following: (A) Cavernosal expression levels of VEGF, catalase, and SOD2 among Mn-treated mice with or without oral B40 I treatment and among the sham mice. (B) Quantified cavernosal VEGF, catalase, and SOD2 levels in Mn-treated mice (Mn, $n=6)$ were significantly lower than those in the sham mice (Sham, $n=6$ ), while they were significantly enhanced under oral B40I treatment $(B 40 I+M n, n=6)$. Values are mean $\pm S E M(* * P<0.0 I$, $* P<0.05$, one-way ANOVA followed by a Student-Newman-Keuls multiple comparisons posttest).

Abbreviations: VEGF, vascular endothelial growth factor; SOD2, superoxide dismutase 2; SEM, standard error of the mean; ANOVA, analysis of variance.

Bax protein determines survival or death after an apoptotic stimulus. We quantified the ratio of Bcl-2/Bax by Western blotting analysis, as shown in Figure 7B. The ratio of Bcl-2/ Bax in the cavernosal tissues of Mn-treated mice under oral B401 treatment had significantly increased compared with that in the Mn-treated mice without oral B401 treatment (Figure 7B, B401+Mn vs Mn, $P<0.01$ ). We further quantified expression levels of Cyt-C by Western blotting analysis. Expression levels of Cyt-C in the cavernosal tissues of
Mn-treated mice under oral B401 treatment were significantly weaker than those of Mn-treated mice without oral B401 treatment (Figure 7B, B401+Mn vs Mn, $P<0.05$ ). Moreover, c-PARP is involved in programmed cell death. ${ }^{21}$ We quantified expression levels of c-PARP by Western blotting analysis. Expression levels of c-PARP in the cavernosal tissues of Mn-treated mice under oral B401 treatment were significantly weaker than those of Mn-treated mice without oral B401 treatment (Figure 7B, B401+Mn vs Mn, $P<0.05$ ).
A

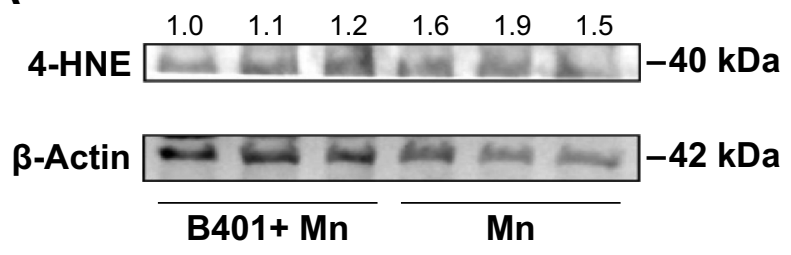

B 4-HNE/ $\beta$-Actin

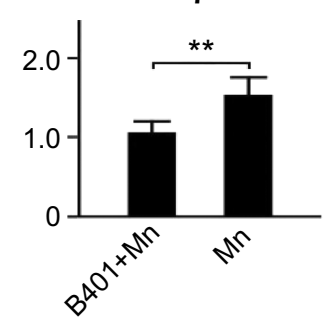

Figure 5 Cavernosal levels of 4-HNE, an inducer of oxidative stress, in Mn-treated mice with and without oral B40I treatment.

Notes: Western blotting analysis shows the following: (A) Cavernosal expression levels of 4-HNE in Mn-treated mice with and without oral B40I treatment. (B) Quantified cavernosal 4-HNE levels of Mn-treated mice under oral B40I treatment (B40I+Mn, n=6) were significantly lower than those in the Mn-treated mice without oral B40I treatment $(M n, n=6)$. Values are mean \pm SEM $(* * P<0.0$ I, one-way ANOVA followed by a Student-Newman-Keuls multiple comparisons posttest). Abbreviations: 4-HNE, 4-hydroxynonenal; SEM, standard error of the mean; ANOVA, analysis of variance. 
A

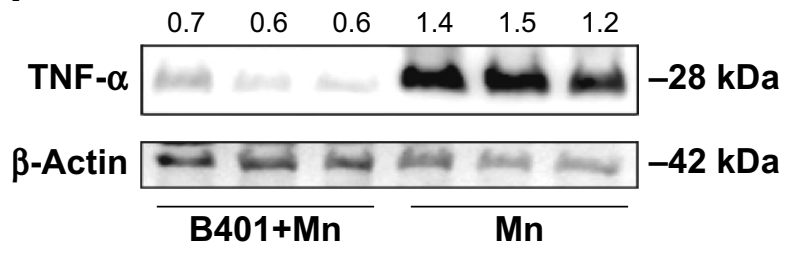

B TNF- $\alpha / \beta-A c t i n$

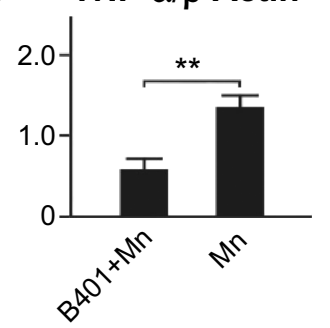

Figure 6 Cavernosal levels of TNF- $\alpha$, a marker of inflammation, in Mn-treated mice with and without oral B40I treatment.

Notes: Western blotting analysis shows the following: (A) Cavernosal expression levels of TNF- $\alpha$ in Mn-treated mice with and without oral B40I treatment. (B) Quantified cavernosal TNF- $\alpha$ levels of Mn-treated mice under oral B40I treatment (B40I+Mn, $n=6$ ) were significantly lower than those in the Mn-treated mice without oral B40I treatment $(\mathrm{Mn}, \mathrm{n}=6)$. Values are mean $\pm \mathrm{SEM}(* * \mathrm{P}<0.0 \mathrm{I}$, one-way ANOVA followed by a Student-Newman-Keuls multiple comparisons posttest).

Abbreviations: TNF- $\alpha$, tumor necrosis factor alpha; SEM, standard error of the mean; ANOVA, analysis of variance.

Apoptosis in the cavernosal tissues of Mn-treated mice was further assessed by estimating the expression levels of caspase-12 and caspase-3, two important activators for apoptosis signaling. We quantified expression levels of caspase- 12 and caspase- 3 by Western blotting analysis, as shown in Figure 8A. Expression levels of both caspase-12 and caspase- 3 in the cavernosal tissues of Mn-treated mice were significantly increased beyond those of the sham mice (Figure $8 \mathrm{~B}, \mathrm{Mn}$ vs Sham, $P<0.01-0.05$ ) but were significantly decreased in the cavernosal tissues of Mn-treated mice under oral B401 treatment (Figure 8B, B401+Mn vs Mn,
$P<0.01-0.05)$. Interestingly, expression levels of caspase- 12 in the cavernosal tissues of Mn-treated mice under oral B401 treatment were significantly higher than those of the sham mice (Figure 8B, B401+Mn vs Sham, $P<0.05$ ).

\section{Discussion}

Erectile dysfunction is a common disorder among aging males. In this study, we evaluated the effects of the Chinese herbal formula B401 in the cavernosal tissues of Mn-treated mice. Our results demonstrated that oral treatment of the herbal formula B401 effectively enhances both synthesis of
A
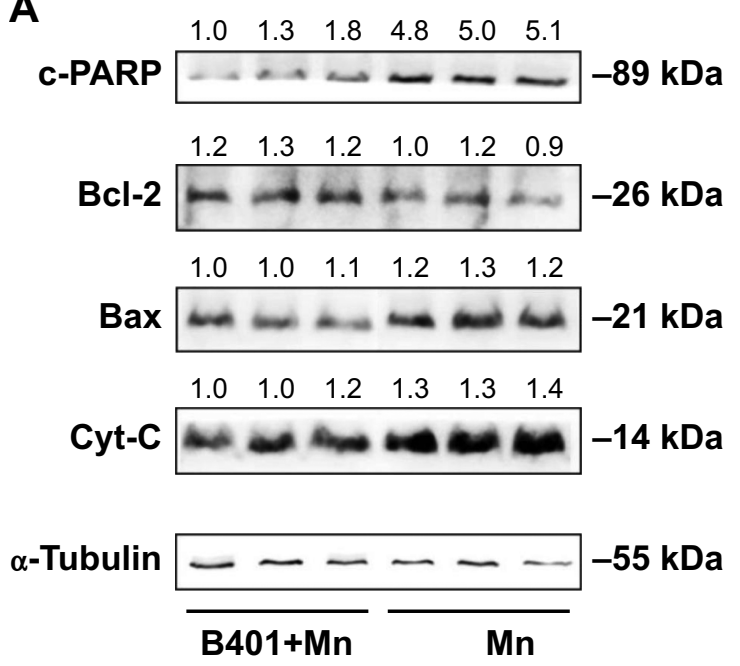

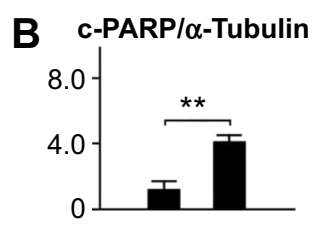

Bcl-2/Bax
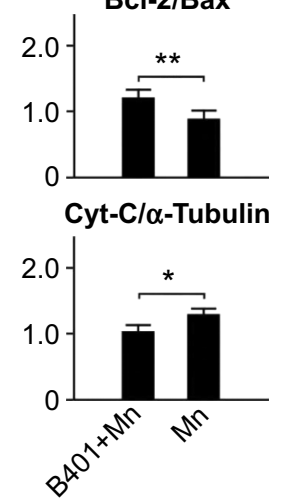

Figure 7 Cavernosal levels of antiapoptotic Bcl-2 and apoptosis inducers c-PARP, Bax, and Cyt-C in Mn-treated mice with and without oral B40I treatment.

Notes: Western blotting analysis shows the following: (A) Cavernosal expression levels of c-PARP, Bcl-2, Bax, and Cyt-C in Mn-treated mice with and without oral B40I treatment. (B) The quantified ratio of cavernosal Bcl-2/Bax in Mn-treated mice under oral B40I treatment (B40I+Mn, $\mathrm{n}=6)$ was significantly enhanced in comparison with the Mn-treated mice without oral B40I treatment (Mn, n=6). Quantified cavernosal c-PARP and Cyt-C levels in Mn-treated mice under oral B40I treatment (B40I+Mn, $n=6$ ) were significantly lower compared with the Mn-treated mice without oral B40I treatment (Mn, $n=6)$. Values are mean $\pm S E M(* * P<0.0$ I, $* P<0.05$, one-way ANOVA followed by a Student-Newman-Keuls multiple comparisons posttest).

Abbreviations: Bcl-2, B-cell lymphoma 2; Bax, Bcl-2-associated X protein; c-PARP, cleaved poly(adenosine diphosphate-ribose) polymerase; Cyt-C, cytochrome c; SEM, standard error of the mean; ANOVA, analysis of variance. 
A

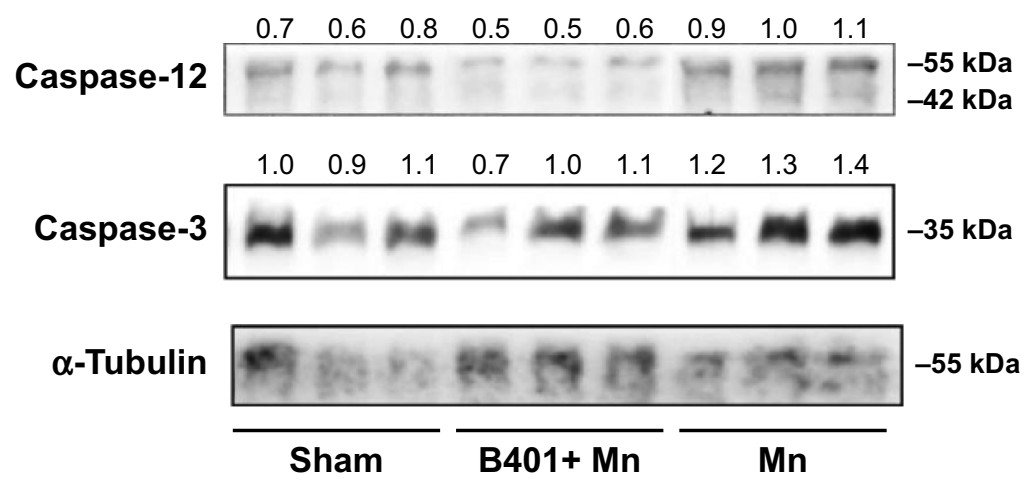

B Caspase-12/ $\alpha$-Tubulin

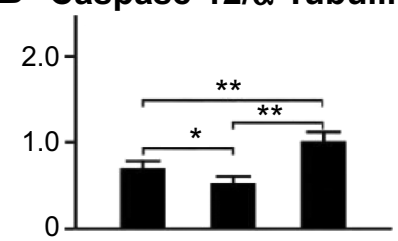

Caspase-3/ $\alpha$-Tubulin

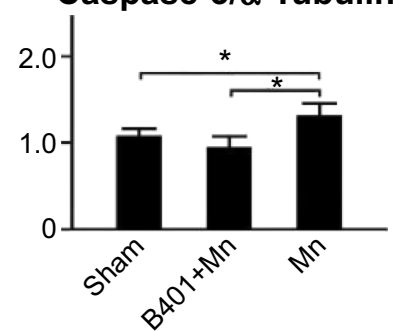

Figure 8 Cavernosal levels of apoptosis inducers caspase-12 and caspase-3 among Mn-treated mice with and without oral B40I treatment, as well as among the sham mice.

Notes: Western blotting analysis shows the following: (A) Cavernosal expression levels of caspase- 12 and caspase-3 among Mn-treated mice with and without oral B40 I treatment, as well as among the sham mice. (B) Quantified cavernosal caspase- 12 and caspase- 3 levels in Mn-treated mice (Mn, $n=6)$ were significantly higher than those in the sham mice (Sham, $n=6$ ), while being significantly reduced under oral B40I treatment (B40I+Mn, $n=6)$. Values are mean \pm SEM (**P $<0.0 \mathrm{I}$, $* P<0.05$, one-way ANOVA followed by a Student-Newman-Keuls multiple comparisons posttest).

Abbreviations: SEM, standard error of the mean; ANOVA, analysis of variance.

nitric oxide (Figures 2 and 3) and angiogenesis (Figure 4), while reducing oxidative stress (Figures 4 and 5), inflammation (Figure 6), and apoptosis (Figures 7 and 8) in the penile corpus cavernosum of Mn-treated mice.

An ingredient of the herbal formula B401 shown in Figure 1, the extract from $P$. ginseng, has been proven to reduce oxidative stress and inflammation. ${ }^{22-25}$ Furthermore, $\mathrm{Rg} 1$ from $P$. ginseng can induce rapid nitric oxide production from eNOS through the phosphatidylinositol 3-kinase $(\mathrm{PI} 3 \mathrm{~K}) /$ protein kinase B (Akt) pathway. ${ }^{26}$ The extract from A. membranaceus has been reported to reduce oxidative stress and enhance nitric oxide production. ${ }^{27,28}$ The extract from $A$. sinensis has been reported to have angiogenic effects in zebrafish through enhancement of VEGF mRNA expression. ${ }^{29}$ The extract from $R$. glutinosa has long been used in age-related diseases and its therapeutic efficacy by attenuating oxidative damage in the brain of mice has been previously reported. ${ }^{30}$ The extract from L. fructus has been used to protect against hydrogen peroxide-induced oxidative damage. ${ }^{31}$ The extract from E. prostrata has been reported to have cerebroprotective and antioxidative effects by reducing global cerebral ischemia in rats. ${ }^{32}$ Taken together, the herbal formula B401 might have protective effects brought about by enhancing production of nitric oxide and VEGF, while reducing oxidative stress and inflammation. As for traditional Chinese medicine, we know that none of these herbs have much therapeutic value. To use any of them alone may prove problematic. That is why we use combined herbal formulas to treat penile toxicity in mice with acute manganism.

As suggested in the Introduction, excessive Mn exposure may lead to erectile dysfunction in human and other animal models. ${ }^{1-6}$ Nitric oxide plays a key role in penile erection. ${ }^{33}$ Impaired nitric oxide formation may be considered a pathological mechanism in erectile dysfunction. ${ }^{34}$ Nitric oxide can be synthesized and released from nonadrenergic, noncholinergic nerve endings by specific nNOS and from the endothelium by eNOS. ${ }^{35}$ It has been suggested that rapid, brief activation of nNOS initiates the erectile process, whereas PI3-kinase/Aktdependent phosphorylation and activation of eNOS by augmented blood flow and endothelial shear stress lead to sustained nitric oxide production and maximal erection. ${ }^{36}$ Inhibition of nNOS attenuates erectile responses. ${ }^{37}$ Thus, it is very likely that physiological penile erection is mediated by both nNOS and eNOS. Furthermore, it has been suggested that VEGF induces penile erection and corrects alterations in eNOS phosphorylation. ${ }^{38}$ Excessive expression of VEGF may induce synthesis of eNOS and iNOS in the penises of rats. ${ }^{39}$ As suggested from our observations in this study, we found that ICR mice had significantly reduced synthesis of nitric oxide (Figure 2) and expression levels of nNOS, eNOS (Figure 3), and VEGF (Figure 4) in penile corpus cavernosum. Taken together, excessive $\mathrm{Mn}$ exposure may lead to erectile dysfunction through inhibition of nitric oxide-mediated signaling pathways. In Taiwan, the Chinese herbal formula B401 has been developed as a health supplement for promoting blood circulation and enhancing 


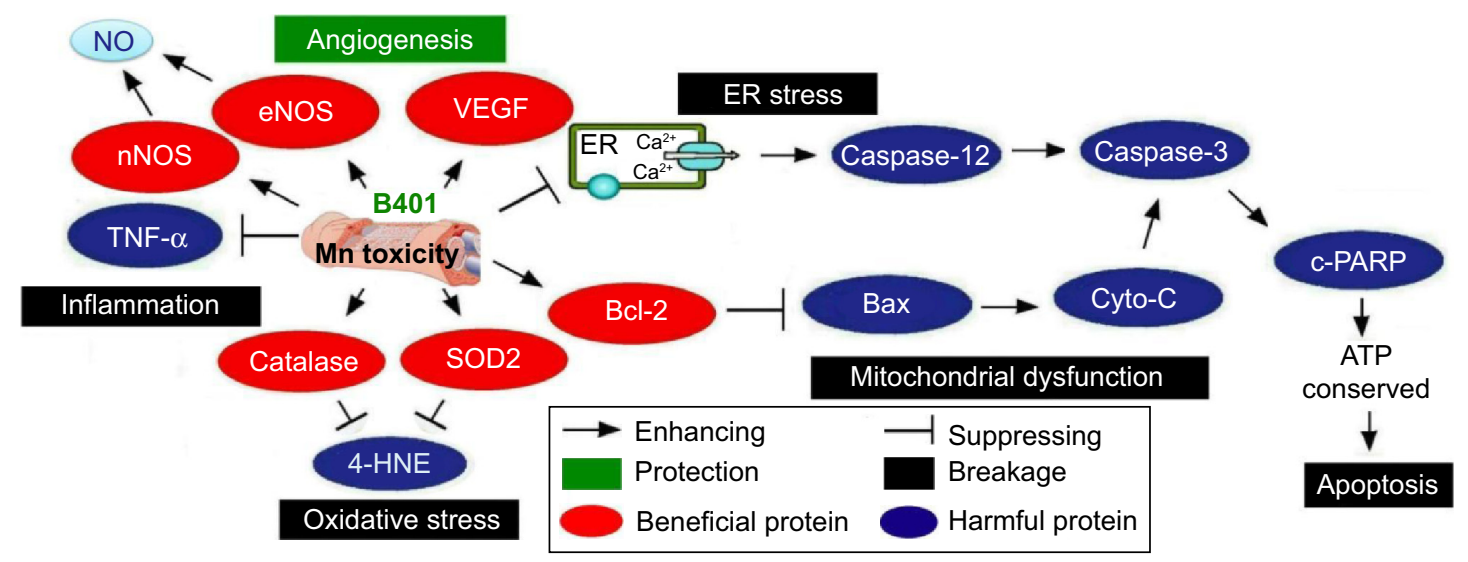

Figure 9 The schematic diagram illustrates that oral B40I treatment may alleviate penile toxicity in Mn-treated mice by enhancing both synthesis of nitric oxide and angiogenesis, while reducing oxidative stress, inflammation, and apoptosis in the corpus cavernosum.

Abbreviations: NO, nitric oxide; nNOS, neuronal nitric oxide synthase; eNOS, endothelial nitric oxide synthase; VEGF, vascular endothelial growth factor; SOD2, superoxide dismutase 2; 4-HNE, 4-hydroxynonenal; TNF- $\alpha$, tumor necrosis factor alpha; ER, endoplasmic reticulum; Bcl-2, B-cell lymphoma 2; Bax, Bcl-2-associated $\mathrm{X}$ protein; c-PARP, cleaved poly(adenosine diphosphate-ribose) polymerase; Cyto-C, cytochrome C; ATP, adenosine triphosphate.

brain function. In this study, we observed that synthesis of nitric oxide and the expression levels of nNOS, eNOS, and VEGF were significantly enhanced in the penile corpus cavernosum of Mn-treated mice under oral treatment with the herbal formula B401 (Figures 2-4). Thus, we assumed that B401 may have protective effects on penile toxicity in mice with acute manganism by promoting nitric oxide-mediated signaling pathways.

Several evidences have indicated that oxidative stress, mitochondrial dysfunction, inflammation, and apoptosis were involved in underlying pathological mechanisms of excessive Mn-induced neurodegeneration. ${ }^{40}$ As suggested from our results, we found that ICR mice had significantly reduced levels of SOD2, catalase (Figure 4), and Bcl-2 (Figure 7) in the penile corpus cavernosum but increased expression levels of 4-HNE (Figure 5), TNF- $\alpha$ (Figure 6), Bax, Cyt-C (Figure 7), caspase-12, and caspase-3 (Figure 8) in the penile corpus cavernosum. Previous studies have suggested that oxidative stress plays a causative role in erectile dysfunction. ${ }^{41,42}$ Enhanced expression of 4-HNE, an oxidative marker, has been observed in a rat model of radiation-induced erectile dysfunction. ${ }^{43}$ Oxidative stress and inflammation lead to endothelium dysfunction, which plays a key part in the pathophysiology of erectile dysfunction. ${ }^{44}$ Administration of the antioxidants catalase and SOD2 may cause diabetes-induced oxidative stress and erectile dysfunction. ${ }^{45}$ Inflammatory conditions were also observed in patients with erectile dysfunction, as shown by increasing plasma levels of TNF- $\alpha .{ }^{46}$ Furthermore, apoptosis is a downstream event in erectile dysfunction. ${ }^{19}$ The Bcl-2 protein plays an important role in inhibiting apoptosis, while Bax is a proapoptotic member that releases Cyt-C from the mitochondria. ${ }^{47,48}$ PARP is cleaved by caspase- 3 and is a key executor of apoptosis. ${ }^{49,50}$ In this study, we observed that expression levels of antioxidative catalase and SOD2, as well as antiapoptotic Bcl-2, were significantly enhanced, but expression levels of 4-HNE, c-PARP, Bax, Cyt-C, caspase-12, and caspase-3 were significantly reduced in the penile corpus cavernosum of Mn-treated mice under oral treatment with the herbal formula B401 (Figures 4-8). In other words, B401 may have protective effects on penile toxicity in ICR mice with acute manganism by reduction of oxidative stress, mitochondrial dysfunction, inflammation, and apoptosis. ICR mice with acute manganism is characterized by reducing nitric oxide-mediated signaling pathways, while increasing oxidative stress, mitochondrial dysfunction, inflammation, and apoptosis in the penile corpus cavernosum of the male reproductive system.

\section{Highlights}

1. We report that excess $\mathrm{Mn}$ treatment increases penile toxicity in aging male mice by reducing nitric oxide synthesis and enhancing oxidative stress, inflammation, and apoptosis in the penile cavernous tissue.

2. Oral treatment with herbal formula B401 alleviates penile toxicity in aging male mice with manganism by promoting nitric oxide synthesis and reducing oxidative stress, inflammation, and apoptosis in penile cavernous tissue.

3. The herbal formula B401 may serve as a potential dietotherapeutic supplement for penile toxicity or dysfunction for aging males.

\section{Conclusion}

As summarized in Figure 9, we have suggested that oral B401 treatment could alleviate penile toxicity in Mn-treated mice 
by enhancing both synthesis of nitric oxide and angiogenesis, while reducing oxidative stress, inflammation, and apoptosis in the corpus cavernosum. It is highly possible that the herbal formula B401 may also be a useful dietotherapeutic supplement for penile toxicity or dysfunction.

\section{Acknowledgments}

The authors acknowledge the Industry-University Cooperative Research program funded by the Brion Research Institute of Taiwan and the National Taiwan Normal University Transnational Research Centers grant (103T3040B04). The funding agency had no role in the study design, data collection and analysis, the decision to publish, or the preparation of the manuscript.

\section{Disclosure}

The authors report no conflicts of interest in this work.

\section{References}

1. Emara AM, El-Ghawabi SH, Madkour OI, el-Samra GH. Chronic manganese poisoning in the dry battery industry. Br J Ind Med. 1971; 28(1):78-82.

2. Mena I, Marin O, Fuenzalida S, Cotzias GC. Chronic manganese poisoning. Clinical picture and manganese turnover. Neurology. 1967; 17(2):128-136.

3. Rodier J. Manganese poisoning in Moroccan miners. $\mathrm{Br} J$ Ind Med. 1995;12(1):21-35.

4. Lauwerys R, Roels H, Genet P, Toussaint G, Bouckaert A, De Cooman S. Fertility of male workers exposed to mercury vapor or to manganese dust: a questionnaire study. Am J Ind Med. 1985;7(2): 171-176.

5. Chandra SV, Ara R, Nagar N, et al. Sterility in experimental manganese toxicity. Acta Biol Med Ger. 1973;30(6):857-862.

6. Seth PK, Nagar N, Husain R, Chandra SV. Effects of manganese on rabbit testes. Environ Physiol Biochem. 1973;3:263-267.

7. Burnett AL, Lowenstein CJ, Bredt DS, Chang TS, Snyder SH. Nitric oxide: a physiologic mediator of penile erection. Science. 1992;257(5068):401-403.

8. Andersson KE, Wagner G. Physiology of penile erection. Physiol Rev. 1995;75(1):191-236.

9. Moncada S. Nitric oxide in the vasculature: physiology and pathophysiology. Ann N Y Acad Sci. 1997;811:60-69.

10. Podlasek CA, Zelner DJ, Bervig TR, Gonzalez CM, McKenna KE, McVary KT. Characterization and localization of nitric oxide synthase isoforms in the BB/WOR diabetic rat. J Urol. 2001;166(2): 746-755.

11. Saenz TI, Goldstein I. Diabetic penile neuropathy. Urol Clin North Am. 1998;15(1):17-22.

12. Sullivan ME, Thompson CS, Dashwood MR, et al. Nitric oxide and penile erection: is erectile dysfunction another manifestation of vascular disease? Cardiovasc Res. 1999;43(3):658-665.

13. Bakircioglu ME, Sievert KD, Nunes L, Lau A, Lin CS, Lue TF. Decreased trabecular smooth muscle and caveolin-1 expression in the penile tissue of aged rats. J Urol. 2001;166(2):734-738.

14. Jiang R, Chen JH, Jin J, Shen W, Li QM. Ultrastructural comparison of penile cavernous tissue between hypertensive and normotensive rats. Int J Impot Res. 2005;17(5):417-423.

15. Rajasekaran M, Kasyan A, Jain A, Kim SW, Monga M. Altered growth factor expression in the aging penis: the Brown-Norway rat model. J Androl. 2002;23(3):393-399.
16. Toblli JE, Stella I, Inserra F, Ferder L, Zeller F, Mazza ON. Morphological changes in cavernous tissue in spontaneously hypertensive rats. Am J Hypertens. 2000;13(6):686-692.

17. Wang SE, Lin CL, Hsu CH, Sheu SJ, Chien CT, Wu CH. Treatment of a herbal formula B401 enhances neuroprotection and angiogenesis in the R6/2 mouse model of Huntington's disease. Drug Des Devel Ther. 2015; 9:887-900.

18. Majid DS, Omoro SA, Chin SY, Navar LG. Intrarenal nitric oxide activity and pressure natriuresis in anesthetized dogs. Hypertension. 1998;32(2):266-272.

19. Yamanaka M, Shirai M, Shiina H, et al. Diabetes induced erectile dysfunction and apoptosis in penile crura are recovered by insulin treatment in rats. J Urol. 2003;170(1):291-297.

20. Machado-Vieira R, Pivovarova NB, Stanika RI, et al. The Bcl-2 gene polymorphism rs956572AA increases inositol 1,4,5-trisphosphate receptor-mediated endoplasmic reticulum calcium release in subjects with bipolar disorder. Biol Psychiatry. 2011;69(4):344-352.

21. Yu SW, Andrabi SA, Wang H, et al. Apoptosis-inducing factor mediates poly(ADP-ribose) polymer-induced cell death. Proc Natl Acad Sci US A. 2006;103(48):18314-18319.

22. Chen XC, Zhu YG, Zhu LA, et al. Ginsenoside Rg1 attenuates dopamine-induced apoptosis in PC12 cells by suppressing oxidative stress. Eur J Pharmacol. 2003;473(1):1-7.

23. Lee JS, Choi HS, Kang SW, et al. Therapeutic effect of Korean red ginseng on inflammatory cytokines in rats with focal cerebral ischemia/ reperfusion injury. Am J Chin Med. 2011;39(1):83-94.

24. Lin WM, Zhang YM, Moldzio R, Rausch WD. Ginsenoside Rd attenuates neuroinflammation of dopaminergic cells in culture. J Neural Transm Suppl. 72:105-112.

25. Yu SC, Li XY. Effect of ginsenoside on IL-1 beta and IL-6mRNA expression in hippocampal neurons in chronic inflammation model of aged rats. Acta Pharmacol Sin. 2000;21(10):915-918.

26. Leung KW, Cheng YK, Mak NK, Chan KK, Fan TP, Wong RN. Signaling pathway of ginsenoside-Rg1 leading to nitric oxide production in endothelial cells. FEBS Lett. 2006;580(13):3211-3216.

27. Ji P, Wei Y, Xue W, et al. Characterization and antioxidative activities of polysaccharide in Chinese angelica and its processed products. Int J Biol Macromol. 2014;67:195-200.

28. Meng L, Qu L, Tang J, Cai SQ, Wang H, Li X. A combination of Chinese herbs, Astragalus membranaceus var mongholicus and Angelica sinensis, enhanced nitric oxide production in obstructed rat kidney. Vascul Pharmacol. 2007;47(2-3):174-183.

29. Lam HW, Lin HC, Lao SC, et al. The angiogenic effects of Angelica sinensis extract on HUVEC in vitro and zebrafish in vivo. $J$ Cell Biochem. 2008;103(1):195-211.

30. Zhang X, Zhang A, Jiang B, Bao Y, Wang J, An L. Further pharmacological evidence of the neuroprotective effect of catalpol from Rehmannia glutinosa. Phytomedicine. 2008;15(6-7):484-490.

31. Ju HY, Chen SC, Wu KJ, et al. Antioxidant phenolic profile from ethyl acetate fraction of Fructus Ligustri Lucidi with protection against hydrogen peroxide-induced oxidative damage in SH-SY5Y cells. Food Chem Toxicol. 2012;50(3-4):492-502.

32. Mansoorali KP, Prakash T, Kotresha D, Prabhu K, Rama Rao N. Cerebroprotective effect of Eclipta alba against global model of cerebral ischemia induced oxidative stress in rats. Phytomedicine. 2012;19(12):1108-1116.

33. Rajfer J, Aronson WJ, Bush PA, Dorey FJ, Ignarro LJ. Nitric oxide as a mediator of relaxation of the corpus cavernosum in response to nonadrenergic, noncholinergic neurotransmission. NEngl J Med. 1992; 326(2):90-94.

34. Kim N, Azadzoi KM, Goldstein I, Saenz de Tejada I. A nitric oxidelike factor mediates nonadrenergic-noncholinergic neurogenic relaxation of penile corpus cavernosum smooth muscle. J Clin Invest. 1991;88(1):112-118.

35. Burnett AL. Nitric oxide in the penis: physiology and pathology. J Urol. 1997;157(1):320-324.

36. Hurt KJ, Musicki B, Palese MA, et al. Akt-dependent phosphorylation of endothelial nitric-oxide synthase mediates penile erection. Proc Natl Acad Sci US A. 2002;99(6):4061-4066. 
37. Ignarro LJ, Bush PA, Buga GM, Wood KS, Fukuto JM, Rajfer J. Nitric oxide and cyclic GMP formation upon electricalfield stimulation cause relaxation of corpus cavernosum smooth muscle. Biochem Biophys Res Commun. 1990;170(2):843-850.

38. Musicki B, Kramer MF, Becker RE, Burnett AL. Age-related changes in phosphorylation of endothelial nitric oxide synthase in the rat penis. J Sex Med. 2005;2(3):347-357.

39. Lin CS, Ho HC, Chen KC, Lin G, Nunes L, Lue TF. Intracavernosal injection of vascular endothelial growth factor induces nitric oxide synthase isoforms. BJU Int. 2002;89(9):955-960.

40. Milatovic D, Zaja-Milatovic S, Gupta RC, Yu Y, Aschner M. Oxidative damage and neurodegeneration in Mn-induced neurotoxicity. Toxicol Appl Pharmacol. 2009;240(2):219-225.

41. Agarwal A, Nandipati KC, Sharma RK, Zippe CD, Raina R. Role of oxidative stress in the pathophysiological mechanism of erectile dysfunction. J Androl. 2006;27(3):335-347.

42. Jeremy JY, Angelini GD, Khan M, et al. Platelets, oxidant stress and erectile dysfunction: an hypothesis. Cardiovasc Res. 2000;46(1):50-54.

43. Kimura M, Rabbani ZN, Zodda AR, et al. Role of oxidative stress in a rat model of radiation-induced erectile dysfunction. J Sex Med. 2012; 9(6):1535-1549.

44. Berk BC, Abe JI, Min W, Surapisitchat J, Yan C. Endothelial atheroprotective and anti-inflammatory mechanisms. Ann N Y Acad Sci. 2001; 947:93-109.
45. Kawakami T, Urakami S, Hirata H, et al. Superoxide dismutase analog (Tempol: 4-hydroxy-2,2,6,6-tetramethylpiperidine 1-oxyl) treatment restores erectile function in diabetes-induced impotence. Int $J$ Impot Res. 2009;21(6):348-355.

46. Giugliano F, Esposito K, Di Palo C, et al. Erectile dysfunction associates with endothelial dysfunction and raised proinflammatory cytokine levels in obese men. J Endocrinol Invest. 2004;27(7):665-669.

47. Jürgensmeier JM, Xie Z, Deveraux Q, Ellerby L, Bredesen D, Reed JC. Bax directly induces release of cytochrome $\mathrm{c}$ from isolated mitochondria. Proc Natl Acad Sci U S A. 1998;95(9):4997-5002.

48. Reed JC. Bcl-2 and the regulation of programmed cell death. $J$ Cell Biol. 1994;124(1-2):1-6.

49. Boulares AH, Yakovlev AG, Ivanova V, et al. Role of poly(ADP-ribose) polymerase (PARP) cleavage in apoptosis. Caspase 3-resistant PARP mutant increases rates of apoptosis in transfected cells. J Biol Chem. 1999;274(33):22932-22940.

50. Jiang C, Wang Z, Ganther H, Lu J. Caspases as key executors of methyl selenium-induced apoptosis (anoikis) of DU-145 prostate cancer cells. Cancer Res. 2001;61(7):3062-3070.
Clinical Interventions in Aging

\section{Publish your work in this journal}

Clinical Interventions in Aging is an international, peer-reviewed journal focusing on evidence-based reports on the value or lack thereof of treatments intended to prevent or delay the onset of maladaptive correlates of aging in human beings. This journal is indexed on PubMed Central, MedLine,

\section{Dovepress}

CAS, Scopus and the Elsevier Bibliographic databases. The manuscript management system is completely online and includes a very quick and fair peer-review system, which is all easy to use. Visit http://www.dovepress. com/testimonials.php to read real quotes from published authors. 